\title{
28
}

\section{The Design of Survivable ATM Networks}

\author{
P.A. Veitch*, D.G. Smith*, and I Hawker** \\ * Communications Division, \\ Department of Electronic and Electrical Engineering, \\ University of Strathclyde, \\ Glasgow G1 1XW, \\ Scotland. \\ ** BT Labs, \\ Martlesham Heath, \\ Ipswich IP5 7RE, \\ England.
}

\begin{abstract}
It is essential that broadband communication networks are survivable. Failure of links or nodes could have disastrous consequences in a very high speed network carrying large volumes of data, for the user, for the service provider and for the network operator. Hence, restoration mechanisms are required which automatically reconfigure routes to avoid the location of a failure. In this work, a backbone ATM mesh network is considered whereby Virtual Paths are cross-connected at special ATM switching nodes. Initially, the means of performing restoration given a certain spare capacity distribution which does not guarantee comprehensive recovery, is examined. This encompasses the preparation required in advance of a failure, and the actual execution of restoration. A network design scheme is subsequently described which enables provisioning of spare capacity for complete restoration from particular failures: the expected Virtual Path traffic demands and routing must be known a priori.
\end{abstract}

Keyword Codes: D.4.4; C.2.1; C.2.2

Keywords: Communications Management; Network Architecture and Design; Network Protocols

\section{Introduction}

Simple and effective restoration techniques will be an essential feature of broadband communications networks to ensure resilience to link or node failures. Service connections which utilise the backbone network must be protected in a cost-effective way. To minimise the damage of a failure, paths which are interrupted must be re-established rapidly. Otherwise, cells, bursts, or actual calls could be dropped, thus seriously degrading the Quality of Service (QoS) provided to subscribers. Those connections which are not directly affected by a failure, must not be disrupted by restoration. In other words, rerouted traffic must not interfere with existing paths so as to affect their performance. 
Restoration is carried out in a mesh network whereby redundant capacity is shared between several possible failures due to the connectivity of the network, whilst protection switching is employed in star or ring networks with $100 \%$ redundancy of protection resources. It is likely that networks of the future will be capable of both functions to ensure survivability [1,2]. This paper concentrates on restoration strategies for ATM core networks configured in a mesh topology.

Due to the logical characteristics of ATM networks based on the Virtual Path (VP) concept, fast reconfiguration of VPs is possible. This is due to the independence of route and capacity allocation in terms of establishing a Virtual Path $[3,4]$. Routing of cells belonging to a VP is carried out at each VP cross-connect with the aid of a unique VP Identifier (VPI) contained in the cell header. The capacity of a VP is reserved when the VP is activated, and managed at the originating node of the path. If the route of the VP was to be changed, different VPIs would be used corresponding to different links traversed by the path. However, no time slot processing is required at intermediate nodes, as would be the case for digital paths in a Synchronous Transfer Mode (STM) network. Faster restoration times are therefore feasible in ATM networks $[5,6,7]$, compared with STM-based SDH networks.

Section 2 reviews the principal features of mesh network restoration techniques, and highlights the concerns which are unique to ATM networks. A pre-assigned path restoration strategy is described in Section 3. The availability of spare capacity will not always be such that all the VPs in the network can be protected by a backup VP. For instance, following restoration from failure, there will be less spare capacity in the network. It is therefore important to configure backup paths in such a way that contention for spare capacity will not occur in the event of a failure. An algorithm which governs the rule of acceptance of a selected VP protection route is proposed. A novel message propagation scheme which should enable fast execution of restoration from link and node failures is also presented, and results of computer simulations are provided to indicate the performance of the scheme. In Section 4, an algorithm for determining the base quantity of spare network capacity which will permit protection from a subset of failures is described. The Virtual Path primary and protection routes, as well as their capacities, must be known $a$ priori. Finally, some conclusions are drawn in Section 5.

\section{Approaches to Restoration}

Prior to a discussion of the details of restoration, some terminology will be introduced. A span is configured between two nodes, and carries a group of optical fibre cables which are terminated at each end node. Working links are active and carry traffic, while spare links are normally idle, but are utilised in the event of failure as part of a restoration scheme. Restoration can be carried out by central or distributed controlling mechanisms. With the former approach, one or more nodes in the network will detect a failure and transmit alarm messages to a centralised operations system over special links. The central unit then uses its global knowledge of the network to decide how to optimally reroute the affected paths, 
and informs the cross-connects accordingly. The cross-connect maps are consequently altered, and traffic is rerouted over alternate paths. A centralised restoration system based on these principles[8] has been developed by AT\&T. Although this approach allows the spare capacity to be utilised optimally, centralised restoration is considered to be too slow to meet the performance demands of broadband networks. Fast recovery from failure is possible by devolving the responsibility of restoration to the network nodes, whereby cross-connect systems exchange messages in a distributed fashion. Restoration with distributed control has been an active area of research since 1987, with the principal application being SDH networks[9, 10, 11].

There are two distinct rerouting alternatives; path restoration (Fig 1(a)) involves the complete reconfiguration of each path which is interrupted by a failure, whereas span restoration (Fig 1(b)) diverts paths around the failed span, whilst preserving the remainder of the original path. Path restoration allows certain node failures to be restored. If the alternate route is selected to be node disjoint from the original, then the failure of any transit nodes along the path can be mitigated. The span restoration technique is often less efficient than path restoration because the restored path is always an elongated version of the original; this is not strictly the case with path restoration. The advantage of span restoration is the speed of execution, since the whole path, which may be long, need not be cleared then re-established.

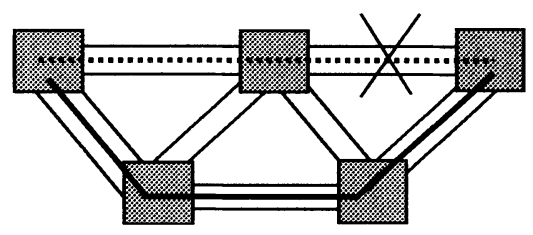

(a) Path restoration

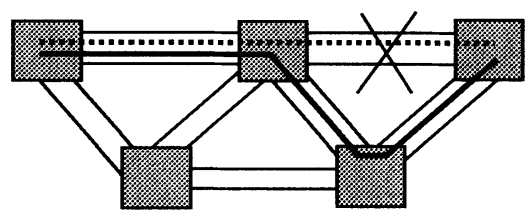

(b) Span restoration

path before failure

restored path

Figure 1: Rerouting from a span failure

Determination of the alternate routes for restoration can be performed either in advance of failure as a contingency measure, or upon detection of a failure, in real time. Pre-assigned 
route restoration techniques (see [12] for SDH, and [5] for ATM applications) typically involve the design of routing tables for each possible single span and single node failure. When a particular failure is recognised, the appropriate routing tables are activated. This approach is costly in terms of storage space and pre-computation burdens on a central manager, and may require synchronisation of the routing table update operation. The advantages of the scheme however, are the ability to make optimal use of resources, and the relative simplicity of execution when compared to dynamic restoration.

Spare capacity design schemes often accompany pre-assigned route restoration strategies $[5$, 6]. For an ATM core network, this requires a knowledge of the routing and bandwidth allocation of Virtual Paths. Using these network characteristics, the amount of spare capacity needed in each span of the network to support restoration from selected failures is computed. If a VP has its route or capacity reconfigured, the current spare capacity allocation would have to be reviewed.

\section{A Virtual Path Restoration Scheme}

\subsection{Feasibility of Protection Virtual Paths}

The scheme presented henceforth combines some of the restoration characteristics described in the previous section. When a VP is established, a central operations system is responsible for setting up an alternate route for the path, to protect it from failure. A path restoration format is favoured so that the path would be restorable if transit nodes fail. It should be noted that if a node fails, all the paths which terminate at that node will be lost. Another reason for favouring path restoration in ATM networks is that path elongation is not as pronounced as in span restoration, so adherence to the bounds on delay and jitter can be retained. This is especially important if VPs are carrying traffic which is predominantly time-sensitive such that processing delays and cell delay variation must be strictly limited.

The requirement of having routing tables to cover all possible failures as is detailed in [12] and [5], is avoided by considering the network state on a path-by-path basis. This results in each path having a single alternative route, regardless of which span/node has failed. Assigning a protection path to a working path is possible due to the logical nature of Virtual Paths whereby routes can be engineered without committing any bandwidth. All that is required to configure a protection VP is the storage of VPIs in routing tables of the appropriate cross-connects (Figure 2).

One-for-one protection paths would not be an economic proposition in restorable STMbased SDH networks, since the reconfiguration occurs at a physical level meaning there would be excessive redundancy of crosspoints and transmission capacity. This is why pre-assigned restoration for SDH networks relies on the replacement of routing tables at certain network nodes to suit a particular failure. With Virtual Paths however, logical capacity reservations are administered meaning more flexible management of spare net- 
work capacity is possible[13]. This is because spare capacity pools can be shared between protection paths activated due to one of many possible failures.

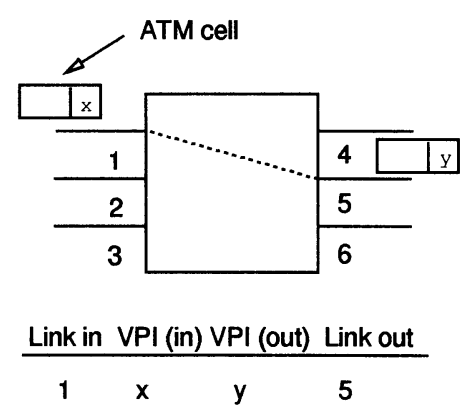

Figure 2: Virtual Path cross-connection

Although flexible sharing of spare capacity is possible with Virtual Path cross-connects, some control must be exercised to ensure that protection paths do not compete for spare capacity during restoration. Thus, a spare capacity contention prevention algorithm is executed for each protection path, prior to VPI configuration at cross-connects. This infers that Virtual Path routes and capacities are not known in advance, such that each time a VP is set up in the network, a centralised system will be responsible for attempting to set up a protection path to be employed for restoration. This protection path has zero bandwidth assignment until it is forced into use due to a failure, and when it is activated, it consumes logical capacity from the appropriate network links.

\subsection{Assigning Protection VPs}

The procedure for protecting VPs across a backbone mesh network will now be described. The central manager has a global view of the network, so it has access to information regarding active path routes and capacities, as well as available spare capacity in the network. Given a VP route, a node disjoint alternate route will be found for the protection path. The criterion for selection here is the shortest path in terms of the number of span hops employed in the route. A decision is then made about whether or not this protection path can be set up with the available spare capacity; this decision is the result of a preplanned contention resolution algorithm.

If the protection path is acceptable, VPIs are loaded into cross-connect routing tables across the path. If the first choice protection path is unacceptable, or if a node disjoint alternate route does not exist because of topological constraints, the manager seeks a span disjoint path instead, based on a shortest hop path requirement. Again, a decision is made on the acceptability of the proposed alternate route, and if the proposal is rejected, the VP is designated unprotected. This process is illustrated in Figure 3. 


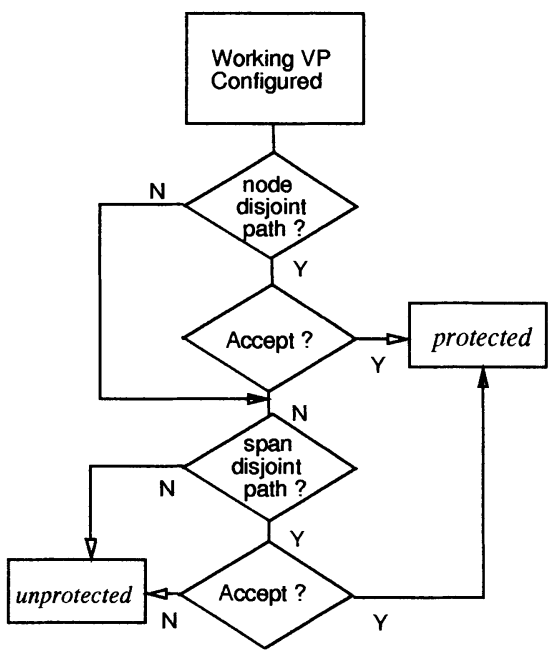

Figure 3: Virtual Path protection algorithm

The algorithm designed to avert spare capacity contention, thus serving as the basis for the decision of acceptance, is presently described.

\subsubsection{Spare Capacity Contention Prevention Algorithm}

Using classical nomenclature, the ATM core network is described as a graph $G(V, E)$, where $V$ is the set of $\mathrm{m}$ vertices, $V=\left\{v_{1}, v_{2}, . ., v_{m}\right\}$, and $E$ is the set of $\mathrm{n}$ edges, $E=$ $\left\{e_{1}, e_{2}, . ., e_{n}\right\}$. A vertex represents an ATM node, and an edge represents a span. It makes matters less complex to consider a span as carrying one (bidirectional) transmission system. This is so that single links can be considered between nodes where every link has a working and spare capacity allocation. Each edge has a quota of spare capacity, so the set of spare capacities is represented by $A=\left\{a_{1}, a_{2}, . ., a_{n}\right\}$. At an arbitrary moment in time, there are $\omega$ (virtual) paths forming a path network $P$, where $P=\left\{p_{1}, p_{2}, . ., p_{\omega}\right\}$. Furthermore, there are $\omega$ protection paths forming a protection path network $P^{\prime}$, where $P^{\prime}=\left\{p_{1}^{\prime}, p_{2}^{\prime}, . ., p_{\omega}^{\prime}\right\}$. If a path $p_{k}$ is unprotected, the corresponding protection path $p_{k}^{\prime}=\emptyset$. Each path is assumed bidirectional, and has an allocated capacity such that $C(P)=$ $\left\{c\left(p_{1}\right), c\left(p_{2}\right), . ., c\left(p_{\omega}\right)\right\}$, fully describes the VP capacities.

Consider an ATM mesh network with the above-stated characteristics. We consider the establishment of VPs to occur at discrete time intervals to simplify the explanation of the algorithm. If the first VP is activated, then so too will be the VP protection scheme. If the VP (labelled $\left.p_{1}\right)$ is configured over edges $(j, k)$, say, and the proposed protection path traverses edges $(n, o, q, r)$, then acceptance will ensue provided $c\left(p_{1}\right)<\min \left\{a_{n}, a_{o}, a_{q}, a_{r}\right\}$. 
Subsequently, if the second VP (labelled $p_{2}$ ) is configured over edges $(i, j)$ with proposed protection path $(l, m, o, p)$ then for single span failure protection, the fact that $\left\{p_{1} \cap p_{2}\right\} \neq \emptyset$ and $\left\{p_{1}^{\prime} \cap p_{2}^{\prime}\right\} \neq \emptyset$, influences the decision of acceptance. If any edge from the set $\left\{p_{1} \cap p_{2}\right\}$ fails, each of the corresponding protection paths will require spare capacity from edges contained in the set $\left\{p_{1}^{\prime} \cap p_{2}^{\prime}\right\}$. The diagram of Figure 4 illustrates the problem.

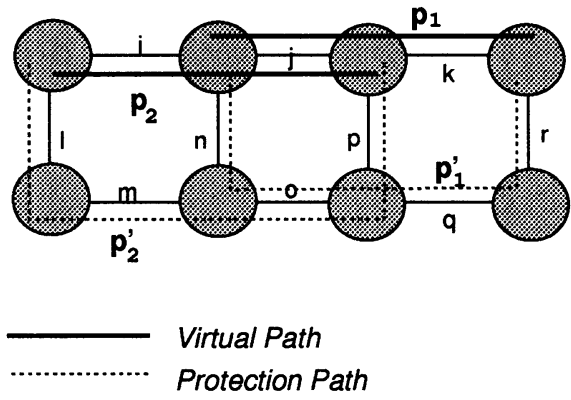

Figure 4: Virtual Paths and proposed protection paths

From the diagram, paths $p_{1}$ and $p_{2}$ overlap on edge $j$, while the protection paths $p_{1}^{\prime}$ and $p_{2}^{\prime}$ overlap on edge $o$. If edge $j$ fails therefore, there would have to be enough spare capacity contained in span $o$ to support both paths. By maintaining a spare capacity availability matrix, VPs are protected only if spare capacity contention is prevented. For protection from single span failures, an $n \times n$ matrix, $S_{n n}$ is required. A matrix element $s_{i j}$, denotes the available spare capacity of edge $e_{j}$ with respect to edge $e_{i}$; the former is a span of the proposed protection path, and the latter is a span of the working VP. Each row of the matrix is initialised with the corresponding spare capacity contained in each edge, such that:

$$
s_{i(1 \leq i \leq n) j}=a_{j} \quad 1 \leq j \leq n
$$

For each edge traversed by a VP, the available spare capacity of each corresponding edge of the proposed protection path is read, and if in any case it is less than the VP capacity, the protection path is deemed unacceptable. If the protection path is accepted the matrix is updated according to the following rule: for every edge of the protection path, $e_{j}$, pertaining to an edge $e_{i}$ of the working path whose capacity is $c(p)$, the following matrix element assignment is made;

$$
s_{i j} \longleftarrow\left(s_{i j}-c(p)\right)
$$

Note that the available spare capacities in the matrix are hypothetical and reflect how the current network state would be altered in the event of restoration from particular 
failures. The algorithm is shown in Figure 5. For convenience, the notation $p\left(E_{x}\right)$ signifies that a path, $p$, traverses $x$ edges, and $p^{\prime}\left(E_{y}\right)$ fully describes the corresponding proposed protection path, $p^{\prime}$, traversing y edges.

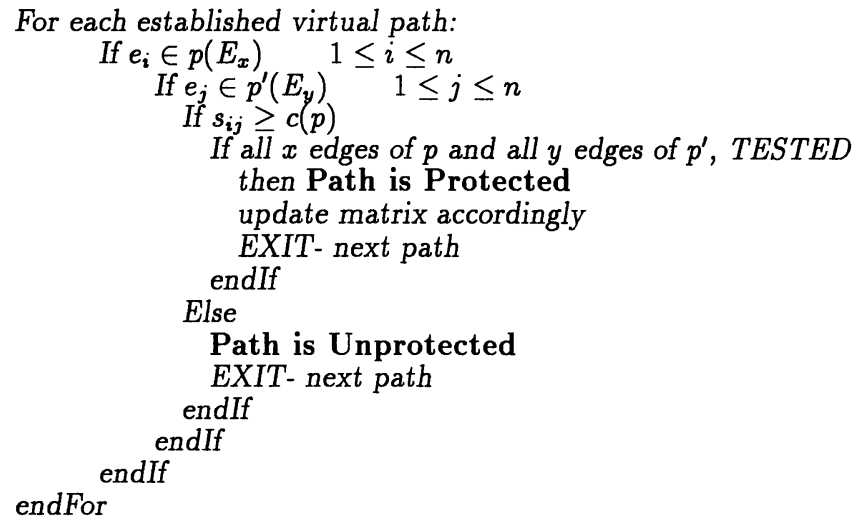

Figure 5: Pre-planned spare capacity contention resolution algorithm

It should be observed that no statistical multiplexing of VPs onto links is enforced, such that the sum of VP capacities for any one link cannot exceed the link capacity. This is a reasonable assumption since ATM networks based on Virtual Paths trade in statistical multiplexing gain for enhanced (simplified) network management capabilities, and simpler call processing. Of course, statistical multiplexing is still possible between VCs multiplexed onto a single VP[14].

\subsubsection{Node Failures}

For transit node failure protection of VPs, the same basic principle of the algorithm of Figure 5 is applied. An $m \times n$ matrix is maintained, $S_{m n}$, and an element $s_{i j}$ would imply the spare capacity which is available in edge $e_{j}$ with respect to vertex $v_{i}$. Hence, for each node traversed by a VP, the available spare capacity per protecting edge is checked against the VP capacity. If the protection path can be supported, the matrix is altered appropriately. Either of the methods may be employed in the overall VP protection program of Figure 3 . Note that if single span failure is the only concern, then the nodedisjoint path search may be omitted.

\subsubsection{Scope of the Scheme}

Although it has been assumed that VPs are established and cleared individually at discrete time intervals, realistically speaking, batch assignment of VPs will also be commonplace. In other words, a network design program may be run to find a suitable path network 
plan to accommodate current traffic demands $[15,16]$. This implies that batch assignment of protection VPs will be required also, and so the algorithm of Figure 5 may well be used to govern such an operation. Given that limited spare capacity exists, the problem is in determining which VPs should be protected. Some prioritisation may have to be arranged based on different classes of calls, and how they would be impacted by failure. Whatever the case, batch assignment of protection paths should be performed with the goal of maximising the number of protected paths.

One scenario which will induce the need for batch path protection is where the network is actually reconfigured (i.e. restored) from a failure. Restoration alters the logical network since VPs are rerouted to divert them from the failure, while the outage itself alters the physical network topology, albeit temporarily. It therefore follows that the spare capacity in the network is diminished in a logical and physical manner; the former is due to restored paths actually utilising spare capacity, and the latter is due to spare capacity associated with the failed span or node being debilitated by the outage. The central control system has to be informed of the new network arrangement so that if a VP is being established while the network is in its reconfigured state, the central manager will not attempt to set up a working VP or protection VP across the failed facilities. Additionally, the new spare capacity distribution must be learned so that further VP protection is possible.

Once the centralised databases have been updated, the VP protection management system must carry out certain tasks to prepare the network for any further failures. VPs that have been recovered no longer have a protection VP since it is employed to replace the original path during restoration. All such VPs are labelled unprotected for the time being. The VPs that are unaffected by a failure, but whose protection VPs cross its path, are also labelled unprotected. The protection scheme is subsequently initiated with as many unprotected VPs being protected from failure as possible.

\subsubsection{An Example Application of the Algorithm}

A sample network was devised with which to apply the protection scheme for both single span and single node failure. Shown in Figure 6, the network is a 20 node mesh.

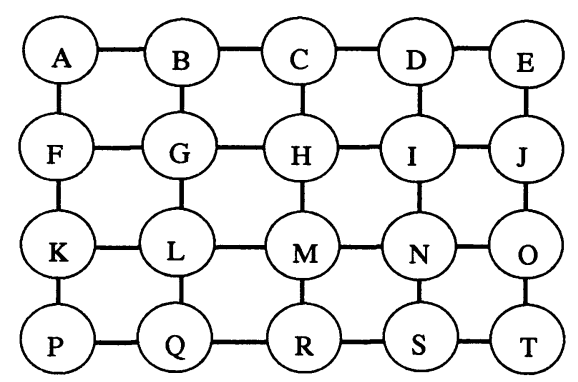

Figure 6: 20 Node ATM mesh network 
There is one VP between each node pair (190 in all), and for simplicity, each VP has unit capacity. No statistical multiplexing of VPs is exercised so that the capacity of each span is simply set at the value required when all the VPs are active. The spare capacity distribution follows a rule of thumb related to the mean valency, of the network. The proportion of spare capacity required per span, in relation to the working capacity, is:

$$
\frac{100}{\nu-1}
$$

For the sample network, where $\nu \approx 3$, the spare capacity per span is fixed at $50 \%$ of the working capacity. A working and protection route are assigned to each VP, with node disjoint paths being selected. For instance, AFGHC is the proposed protection path for the VP between nodes A and C, routed over ABC. The aim is to take each VP in turn and using the proposed protection path, determine whether or not the VP is protected. The $V P$ protection ratio, $\rho$, which is the ratio of protected VPs to all network VPs, is therefore found. It should be emphasised that the spare capacity provisioning is intentionally chosen such that full protection is not guaranteed, because realistically, this will sometimes be the case. For the single span failure protection scheme, $\rho=0.863$, and for single node failure protection, $\rho=0.6474$. The low value for node failure protection is not unexpected since the provisioning rule employed is for dealing principally with single span failures.

For the sample network configuration, 26 VPs would be unprotected from single span failure, using the proposed scheme. Recalling that a VP will not be protected if at least one of the spans of the proposed protection path has insufficient available spare capacity, then there will often be spare capacity remaining in the network. Any VPs remaining unprotected could thus be restored by dynamic mechanisms. The development of novel dynamic route search techniques is an ongoing element of this study, but is outwith the scope of this paper. This section dealt principally with the preparation required for implementing path restoration. The actual means of performing restoration in response to single span and single node failures will now be considered.

\subsection{Path Restoration With Distributed Control}

\subsubsection{A Novel Technique}

As a consequence of the management operations described in the preceding section, VPIs will be written into the appropriate cross-connect systems representing protection VPs. At the source of a protected VP, working and protection entries will exist, with the working entry being active under normal circumstances. To execute restoration of a VP, its working entry must be disabled, and the protection entry enabled. This paper proposes a novel technique to execute path restoration. The method adopted by Kawamura et al[6] involves detection of a failure at the downstream end of the path, and subsequently sending a restoration message over the route of the backup VP. This is shown in Figure 7(a). 


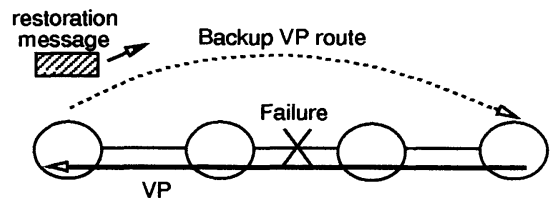

(a)

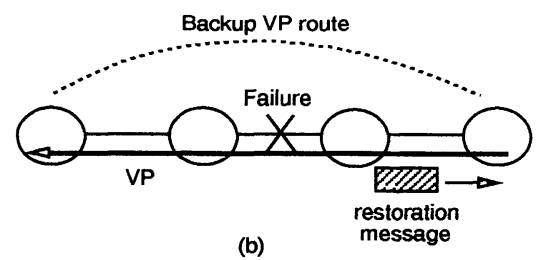

Figure 7: Distributed message relay

Since bidirectional transmission plant exists within a span, each node adjacent to the failure will detect the syndrome. It is therefore suggested that a restoration message be sent to the source of the VP over the working VP route, for each VP that has failed (Figure $7(\mathrm{~b})$ ). Each node that detects a failure determines which incoming VPs are routed over the failed span. A message is backtracked over each VP route. At the origin of each VP, the routing tables are altered so that protection VPs are activated, and traffic is subsequently routed over the protection path. Figure 8 shows an example of the procedure.

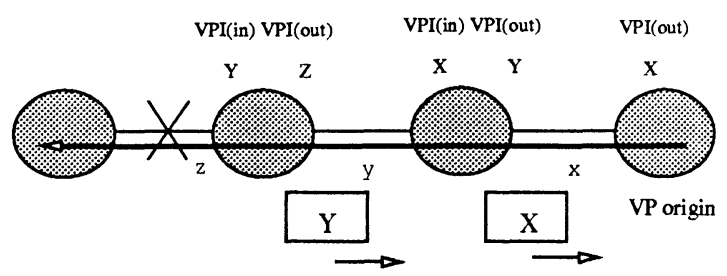

Figure 8: Restoration message alteration per node

For the single span failure illustrated, the upstream node detects the physical layer failure since it is assumed that there is bothway information flow, though not necessarily in a symmetric fashion. It is quite feasible to monitor alarms at the physical layer and take subsequent action at the ATM layer by way of appropriate Operations, Administration and Maintenance (OAM) cell transfer[17]. The node looks for the VPs routed over the failed span, and writes the corresponding VPI(in) into the restoration message, which is routed over the span upon which the VP arrives at the node. At the next upstream node, the VPI is read from the message, and the node locates the VPI(in) whose VPI(out) 
corresponds to that of the restoration message; this VPI is written into the message (over the previous one), and the message is forwarded over the relevant span. When the VP originator receives the restoration message, the VPI which it contains designates which VP entry has to have its working (VPI(out), link(out)) tuple replaced by the corresponding protection tuple. The restoration can be completed very quickly, since fewer nodes will be traversed by the restoration messages than in the scheme of [6]. Furthermore, multiple VPIs may be mapped onto a single OAM cell which backtracks across a particular link. The backtracking function is possible with unidirectional VPs, hence does not rely on bidirectional Virtual Paths. This could be a distinct advantage in managing asymmetric broadband networks[18].

It is assumed that node failures are equivalent to multiple span failures, so that several nodes detect the failure and initiate restoration message transmission. This is shown in Figure 9.

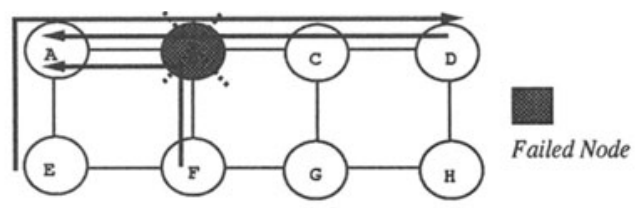

a) Node B Fails

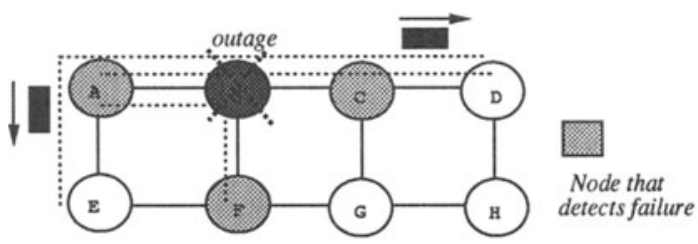

b) Message propagation
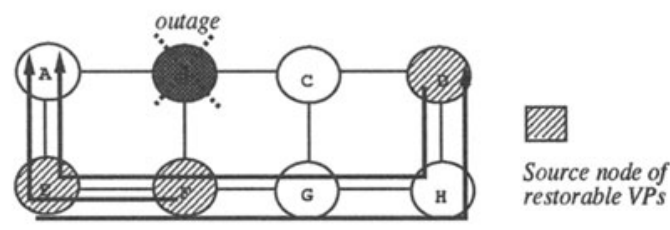

c) Restoration complete restorable VPs

Figure 9: Executing node failure restoration

In part a) of the illustration, node B of the mesh is shown to fail. Many VPs are set up on the network, but only three of interest are shown: these are VPs which traverse node B as 
a transit node. The node disjoint backup VPs are not shown, but are specified as FEA for path FBA, DHGFEA for DCBA, and EFGHD for EABCD. In part b) of the figure, the failure is shown to be detected by nodes $\mathrm{A}, \mathrm{F}$, and $\mathrm{C}$. As mentioned earlier, this is possible as long as each span carries at least one VP in either direction of transmission. Nodes A and $\mathrm{C}$ send a restoration message towards the origin of those VPs which are routed in the direction of the failure. Since node F happens to be the source node of the VP which is routed over FBA, no message propagation is required. This VP will therefore be restored (i.e. switched from the working to the protection route) rapidly. Part c) of the diagram depicts the network configuration following restoration of all the Virtual Paths affected by the node failure.

\subsubsection{Simulation Results}

The new restoration scheme was modelled and simulated to compare it with the existing technique. The network model consisted of $20 \mathrm{VP}$ cross-connects in a mesh (Figure 6), with 190 VPs in all. For the simulation study, VPs were designated to be unidirectional. Separate simulations were run for single span failures, where 164 paths are protected, and single node failures, where 123 paths are protected. The transmission delays were for $155 \mathrm{Mbit} / \mathrm{s}$ links, propagation delays were varied from 0.01 to $0.15 \mathrm{msec}$, and the cell processing time was set at $1 \mathrm{msec}$. These rather basic assumptions were made to facilitate a direct performance comparison in terms of restoration speed. Figure 10 shows the mean restoration ratio versus time following failure detection for each span failure.

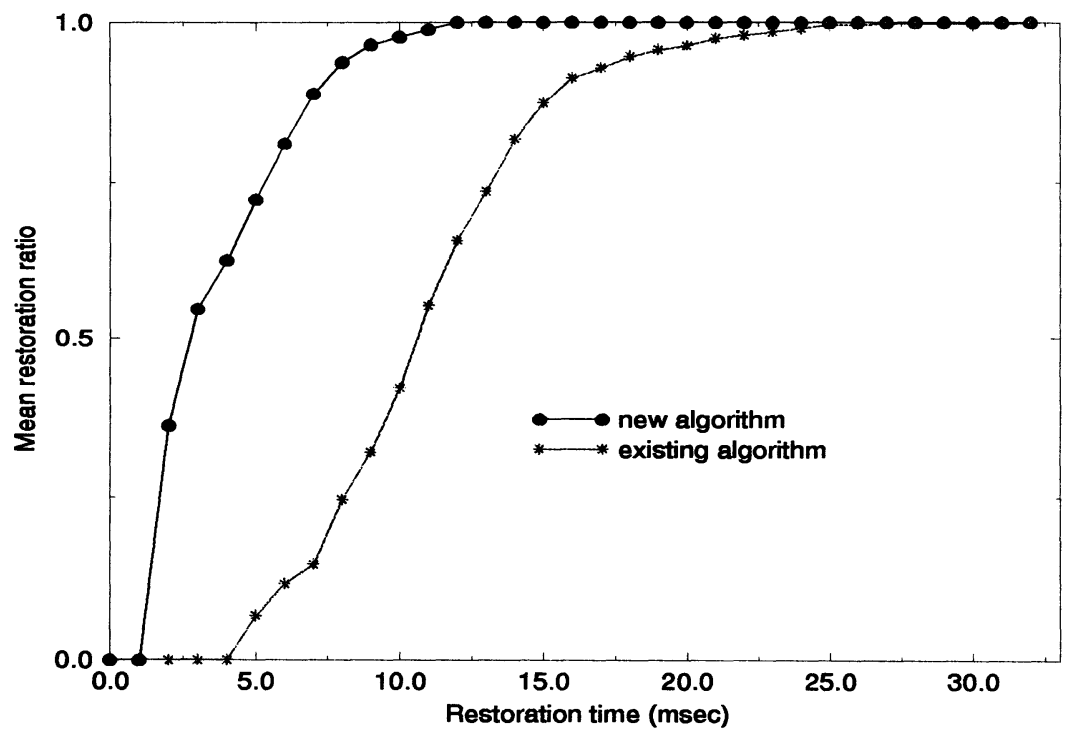

Figure 10: Mean results for 31 span failures 
Figure 11 shows the results for single node failures. It is important to note that the restoration ratio plotted is the ratio of the number of restored VPs to the number of protected VPs that fail; this is why the value converges to 1 . For pre-assigned restoration, the interest lies in the speed of recovery of protected paths. Any unprotected paths which fail are the concern of the dynamic restoration process. The graphs demonstrate the possible speed improvement that can be gained with the new backtracking message relay scheme.

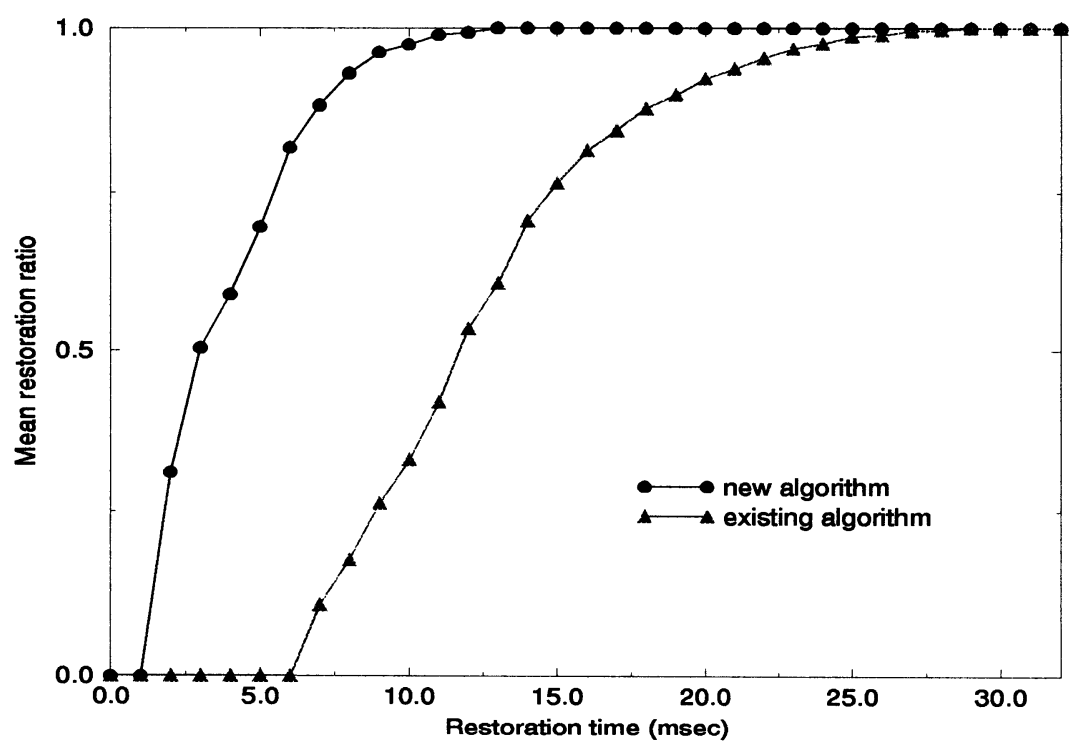

Figure 11: Mean results for 20 node failures

\subsection{Discussion}

The preceding discussion encompasses the planning and execution of Virtual Path restoration in an ATM network. It was assumed that spare capacity exists in the network, but that there is no guarantee of complete VP coverage from failure. The planning algorithm described attempts to protect as many VPs as possible from single span or single node failure, thus ensuring fast restoration without contention for spare capacity. The protection mechanism could be further incorporated into an optimisation program which tries many combinations of working and protection path arrangements to arrive at a global maximum in terms of the number of paths protected. If VPs remain unprotected, it may be possible to restore them dynamically by utilising any remaining spare capacity from the primary restoration phase. The final section proposes a simple spare capacity design algorithm. For a set network configuration, the spare capacity distribution required for total VP protection from certain failures is calculated. 


\section{A Spare Capacity Design Algorithm}

Given a particular network design, with the routes and capacity of each VP and its backup specified in advance, the quantity of spare capacity required in each span to permit restoration from single span failure may be computed using a simple algorithm. In essence, the technique works in reverse to that for spare capacity contention resolution. We define an $n \times n$ spare capacity provisioning matrix, $Q_{n n}$, with an element $q_{i j}$ denoting the required spare capacity in edge $e_{j}$ with respect to edge $e_{i}$. All the elements of the matrix are initiated at zero. The required spare capacity can be found from the provisioning matrix when all paths have been considered. For any edge, $e_{j}$, the required spare capacity, $a_{j}$, will be the largest numerical entry in the corresponding column of the matrix. The algorithm is given in Figure 12.

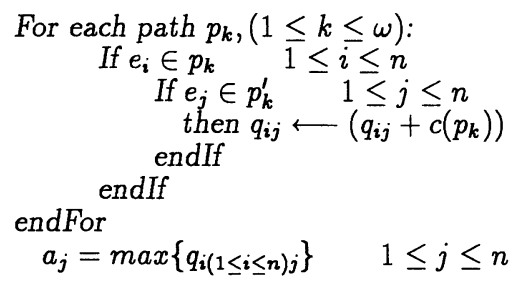

Figure 12: Spare capacity design algorithm

For protection from single node failure, the same principle applies, with an $m \times n$ provisioning matrix, $Q_{m n}$ being generated. Both algorithms were applied to the 20 node network described earlier, and the spare capacity required in the network, expressed as a percentage of the total working capacity, was found to be $52.4 \%$ for the single span failure case, and $70.5 \%$ for the single node failure case. This is a small increase over that of the crude provisioning rule adopted earlier, yet full protection is achieved $(\rho=100 \%)$. This is because spare capacity is deployed only where needed. Note that the measures of spare capacity described are precise logical capacities. Due to modular physical provisioning, the actual capacity required will probably increase. It is stressed however, that the result of the spare capacity calculation was for a fixed path and protection path network design: the spare capacity requirement could undoubtedly be reduced by a combinatorial search of all possible configurations of the paths and their protection routes.

The whole question of spare capacity deployment is vitally important with regard to the efficient and effective management of survivable networks. Since ATM networks are expected to permit flexible bandwidth allocation and control, this advantage must not be lost when provisioning spare resources for the purpose of restoration. 


\section{Conclusion}

This paper has addressed some important issues concerning ATM network survivability. Given a fixed spare capacity distribution in a mesh network, an algorithm was proposed for Virtual Path protection. Either single span or single node failures are accounted for. A centralised system finds an alternate route for a VP following configuration, and crossconnect tables will be set up provided enough spare capacity would be available for the new VP and current VPs, in the event of a failure. Otherwise, a VP remains unprotected, and dynamic restoration may be required. The actual execution of restoration of protected paths is by the distributed control of cross-connect systems in the network. A novel message relay scheme was proposed and the results of a computer simulation suggest that fast restoration times are possible. Some issues remaining for study are OAM cell formatting, and the nodal architecture required to support the backtracking algorithm.

Although dynamic mechanisms have been suggested for use when VPs cannot be protected, they still merit further investigation as stand alone restoration techniques. This is because, when exploited as part of a distributed network control scheme, the operation is carried out autonomously in the network. As a result there is independence from a centralised, and therefore vulnerable, computer system.

A spare capacity design algorithm was explained which provides the minimum logical capacity requirements necessary for complete recovery of VPs from single span failure; the ideas easily extend to single node failure coverage. The VP routes, capacities, and backup routes must be known prior to the computation. If the VP network, $P$, is reconfigured, the spare capacity requirement will change, and $100 \%$ protection (and thus restorability) is no longer guaranteed. This is especially true if the reconfiguration is the outcome of restoration from failure since physical spare capacity will be utilised, diminishing the availability. This is why the preparation strategy, incorporating the spare capacity contention resolution algorithm, is essential for the management of survivable reconfigurable ATM networks.

\section{References}

[1] GROVER, W.D. Case Studies of Survivable Ring, Mesh and Mesh-Arc Hybrid Networks. In IEEE Global Telecommunications Conference (Globecom '92), Orlando, Florida, volume 1, pages 633-638, Dec 6-9 1992.

[2] WU, T-H. Fibre Network Service Survivability. Artech House, 1992.

[3] SATO, K-I., OHTA, S., and TOKIZAWA, I. Broadband ATM Network Architecture Based on Virtual Paths. IEEE Transactions on Communications, 38(8):1212-1222, August 1990. 
[4] SATO, K-I. and TOKIZAWA, I. Flexible Asynchronous Transfer Mode Networks Utilizing Virtual Paths. In IEEE International Conference on Communications (ICC '90), pages 0831-0838, April 1990.

[5] ANDERSON, J., DOSHI, B.T., DRAVIDA, S., and HARSHAVARDHANA, P. Fast Restoration of ATM Networks. IEEE Journal on Selected Areas in Communications, 12(1):128 - 138, January 1994.

[6] KAWAMURA, R., SATO, K-I., and TOKIZAWA, I. Self-Healing ATM Networks Based on Virtual Path Concept. IEEE Journal on Selected Areas in Communications, 12(1):120 - 127, January 1994.

[7] FUJII, H. and YOSHIKAI, N. Restoration Message Transfer Mechanism and Restoration Characteristics of Double-Search Self-Healing ATM Network. IEEE Journal on Selected Areas in Communications, 12(1):149 - 157, January 1994.

[8] FALCONER, W.E. Service Assurance in Modern Telecommunications Networks. IEEE Communications Magazine Special Issue- Surviving Disaster, 28(6):32-39, June 1990.

[9] GROVER, W. D. The SelfHealing Network: A Fast Distributed Restoration Technique for Networks using Digital Cross-connect Machines. In IEEE Global Telecommunications Conference 1987 (Globecom '87), Tokyo, Japan, pages 10901095, November 1987.

[10] YANG, H. C. and HASEGAWA, S. FITNESS- Failure Immunization Technology for NEtwork Service Survivability. In IEEE Global Telecommunications Conference 1988 (Globecom '88), Ft. Lauderdale, Florida, pages 1549-1554, December 1988.

[11] CHOW, C.E., BICKNELL, J.D., McCAUGHEY, S., and SYED, S. A Fast Distributed Network Restoration Algorithm. In IEEE 12th International Conference on Computers and Communications, Phoenix, AZ, pages 261 - 267, March 1993.

[12] COAN, B.A., VECCHI, M.P., and WU, L.T. A Distributed Protocol to Improve the Survivability of Trunk Networks. In XIII International Switching Symposium, Stockholm, Sweden, volume IV, pages 173-179, May 1990.

[13] SATO, K-I., HADAMA, H., and TOKIZAWA, I. Network Reliability Enhancement with Virtual Path Strategy. In IEEE Global Telecommunications Conference (Globecom '90), San Diego, California, pages 403.5.1-403.5.6, December 1990.

[14] HUGHES, D. and WAJDA, K. Comparison of Virtual Path Bandwidth Assignment and Routing Methods. Annales des Telecommunications, 49(1-2):80-89, 1994.

[15] LOGOTHETIS, M. and SHIODA, S. Centralized Virtual Path Bandwidth Allocation Scheme for ATM Networks. IEICE Trans. Commun, E-75B(10):1071-1080, October 1992. 
[16] ARVIDSSON, A. High Level B-ISDN/ATM Traffic Management in Real Time. In Proc 2nd Workshop on Performance Modelling and Evaluation of ATM Networks, Bradford, England, pages 10/1-10/12, July 1994.

[17] BREUER, H-J. ATM Layer OAM: Principles and Open Issues. IEEE Communications Magazine, 29(9):75-78, September 1991.

[18] OHTA, S. and FUJII, N. Applying OSI Systems Management Standards to Virtual Path Testing in ATM Networks. In Proc. IFIP TC6/WG6.6 3rd Int. Symposium on Integrated Network Management, pages 629-640, April 1993. 